\title{
Optimized Retinal Nerve Fiber Layer Segmentation Based on Optical Reflectivity and Birefringence for Polarization-Sensitive Optical Coherence Tomography
}

\author{
Bingqing Wang ${ }^{\mathrm{a}}$, Amit S. Paranjape ${ }^{\mathrm{a}}$, Biwei Yin ${ }^{\mathrm{b}}$, Shuang Liu ${ }^{\mathrm{a}}$, \\ Mia K. Markey ${ }^{\mathrm{a}}$, Thomas E. Milner ${ }^{\mathrm{a}}$, and H. Grady Rylander III*a \\ ${ }^{a}$ Dept. of Biomedical Engineering, The University of Texas at Austin, \\ 1 University Station C0800, Austin, TX, USA 78712-1084 \\ ${ }^{b}$ Dept. of Electrical and Computer Engineering, The University of Texas at Austin, \\ 1 University Station C0803, Austin, TX, USA 78712-1084
}

\begin{abstract}
Segmentation of the retinal nerve fiber layer (RNFL) from swept source polarization-sensitive optical coherence tomography (SS-PSOCT) images is required to determine RNFL thickness and calculate birefringence. Traditional RNFL segmentation methods based on image processing and boundary detection algorithms utilize only optical reflectivity contrast information, which is strongly affected by speckle noise. We present a novel approach to segment the retinal nerve fiber layer (RNFL) using SS-PSOCT images including both optical reflectivity and phase retardation information. The RNFL anterior boundary is detected based on optical reflectivity change due to refractive index difference between the vitreous and inner limiting membrane. The posterior boundary of the RNFL is a transition zone composed of birefringent axons extending from retinal ganglion cells and may be detected by a change in birefringence. A posterior boundary detection method is presented that segments the RNFL by minimizing the uncertainty of RNFL birefringence determined by a Levenberg-Marquardt nonlinear fitting algorithm. Clinical results from a healthy volunteer show that the proposed segmentation method estimates RNFL birefringence and phase retardation with lower uncertainty and higher continuity than traditional intensity-based approaches.
\end{abstract}

Keywords: optical coherence tomography; retinal imaging; retinal nerve fiber layer segmentation

\section{INTRODUCTION}

Optical coherence tomography (OCT) provides real time non-invasive cross-sectional images by measuring the backscattered light ${ }^{1}$. As a functional extension of OCT, polarization-sensitive OCT (PSOCT) is capable of characterizing the polarimetric properties of a birefringent sample including form-birefringence, phase retardation and optical-axis orientation, by measuring the interference fringe intensity and relative phase delay of two orthogonal polarization states ${ }^{2-4}$.

In recent years, PSOCT has found promising application in detecting early stage glaucoma by measuring the thickness and birefringence of the retinal nerve fiber layer $(\mathrm{RNFL})^{5-9}$. Compared with normal eyes, RNFL thickness and birefringence both decrease in glaucoma eyes ${ }^{7,8}$. Previous studies show that RNFL birefringence is mainly derived from the microtubules within retinal ganglion cells (RGCs) ${ }^{10}$. With appropriate polarimetric models ${ }^{11,12}$ and carefully defined RNFL boundaries, RNFL birefringence, which provides functional information of microtubule density, can be recovered from PSOCT data ${ }^{5,13}$.

Segmentation of the RNFL from PSOCT images is fundamental to determine RNFL thickness and birefringence. PSOCT polarimetric processing algorithms are sensitive to slight misdetection of RNFL boundaries. Various sophisticated RNFL segmentation approaches have been reported ${ }^{14-19}$. Mujat et al. ${ }^{14}$ used a deformable spline algorithm to determine RNFL thickness. Garvin et al ${ }^{15}$ presented a 3-D graph search approach for OCT retinal layer segmentation. Paranjape et al. ${ }^{16}$ introduced a simple and fast segmentation method based on a Sobel filter for automated RNFL boundary detection. Mishra et al. ${ }^{17}$ developed a two-step scheme which first identifies the approximate locations of the

*rylander@mail.utexas.edu; phone 1-512-471-1995

Applications of Digital Image Processing XXXIV, edited by Andrew G. Tescher, Proc. of SPIE Vol. 8135, 81351R · @ 2011 SPIE · CCC code: $0277-786$ X/11/\$18 · doi: $10.1117 / 12.892826$ 
RNFL and then performs a local kernel-based optimization to refine segmentation results. The algorithm was successfully applied on OCT scans of rodent eyes. Kajić et al. ${ }^{18}$ utilized texture and shape features of OCT images to detect RNFL boundary. This approach was proven to be insensitive to noise in OCT human retinal images. Mayer et $a l .{ }^{19}$ designed a segmentation approach based on the minimization of an energy function consisting of gradient and local smoothing terms, and evaluated the algorithm on both normal and glaucoma eyes. All of these approaches provide promising RNFL segmentation results. However, none are optimized for estimation of RNFL birefringence using PSOCT for several reasons. First, these algorithms are designed for continuous ring scan or raster scanning patterns, rather than a discrete clustered ring scanning pattern currently employed in PSOCT systems required to minimize degrading effects of speckle noise in polarimetric signals. Second, all of these methods utilize only OCT intensity information of backscattered light, which is strongly corrupted by speckle noise. No reported method takes advantage of functional birefringence information provided by PSOCT. Third, unlike the anterior RNFL boundary where change of refractive index from vitreous to the inner limiting membrane is abrupt, the posterior RNFL boundary is a transition zone from the birefringent RGC axons to their cell bodies; thus, a physiologically well-defined posterior RNFL boundary does not exist in OCT intensity images. The polarimetric measurement capability of PSOCT provides additional information to potentially overcome the limitations of current RNFL segmentation approaches for birefringence measurement.

Utilizing both RNFL intensity and polarimetric information, we propose a two-step RNFL segmentation method for data recorded from swept source polarization-sensitive optical coherence tomography (SS-PSOCT) to achieve optimized RNFL segmentation for birefringence estimation. RNFL boundaries are first estimated from the intensity images using a simple filter followed by thresholding approach. Optimized RNFL segmentation is then achieved by locally moving the estimated RNFL posterior boundary and minimizing uncertainty of estimated RNFL birefringence determined by the Levenberg-Marquardt algorithm. This method segments the RNFL with optimized RNFL birefringence estimation. Performance of the segmentation approach is evaluated using clinical data from a healthy volunteer (NCT\# 01222065).

\section{METHODS}

\subsection{SS-PSOCT data acquisition}

The clinical SS-PSOCT system is similar to a previously described system ${ }^{5}$ except the $50 / 50$ coupler in detection arm is replaced with a 50/50 bulk-optic beam splitter. Briefly, the SS-PSOCT swept source emits at a $1059 \mathrm{~nm}$ center wavelength with $58 \mathrm{~nm}$ full-width-half-max (FWHM) spectral bandwidth and $27 \mathrm{kHz}$ sweep repetition rate. The polarization state of the incident beam input into the interferometer is adjustable with an electro-optic modulator. The detection arm features a bulk-optic polarization sensitive balanced detection module to cancel noise and record interference fringe signals in two orthogonal polarization states. The SS-PSOCT system provides $12 \mu \mathrm{m}$ axial resolution in the RNFL.

During SS-PSOCT measurements, two retinal scanning patterns are employed: a raster scan and a clustered ring scan. The raster scan is used to record the pattern of blood vessels on the retina. A raster scan is comprised of 500 A-lines per $\mathrm{B}$-scan and $160 \mathrm{~B}$-scans that cover a $6 \mathrm{~mm} \times 6 \mathrm{~mm}$ square area centered on the optic nerve head. The clustered ring scan is used to acquire intensity and polarimetric data. The clustered ring-scan pattern (Fig. 1) consists of 10 concentric rings around the optic nerve head with $2 \mathrm{~mm}-5 \mathrm{~mm}$ diameters, and each ring contains 36 clusters of $100 \mathrm{~A}$-lines. In each cluster the 100 A-lines are acquired by sampling a sinusoidal pattern of 5 periods. For each A-line, depth-resolved data are recorded with three input polarization states controlled by an electro-optic modulator $\left(\mathrm{S}_{0}:-2 \pi / 3, \mathrm{~S}_{1}: 0\right.$, and $\mathrm{S}_{2}: 2 \pi / 3$ which at the output of the electro-optic modulator are equally spaced on the QU plane of the Poincaré Sphere). The 100 A-lines in each cluster are assumed to be uncorrelated and sample nearly identical polarimetric properties since they are acquired over a relatively small spatial region. Averaging $N_{A}=100$ uncorrelated A-lines increases polarization signal-tonoise ratio (PSNR) by approximately $\sqrt{N_{A}}=10$ times $^{20}$.

Clinical data are acquired from a healthy volunteer's right (OD) eye at Eye Institute of Austin. Imaging protocols have been approved by the Institutional Review Board of the University of Texas at Austin (IRB protocol \# 2007040101) and are registered (NCT \# 01222065). 


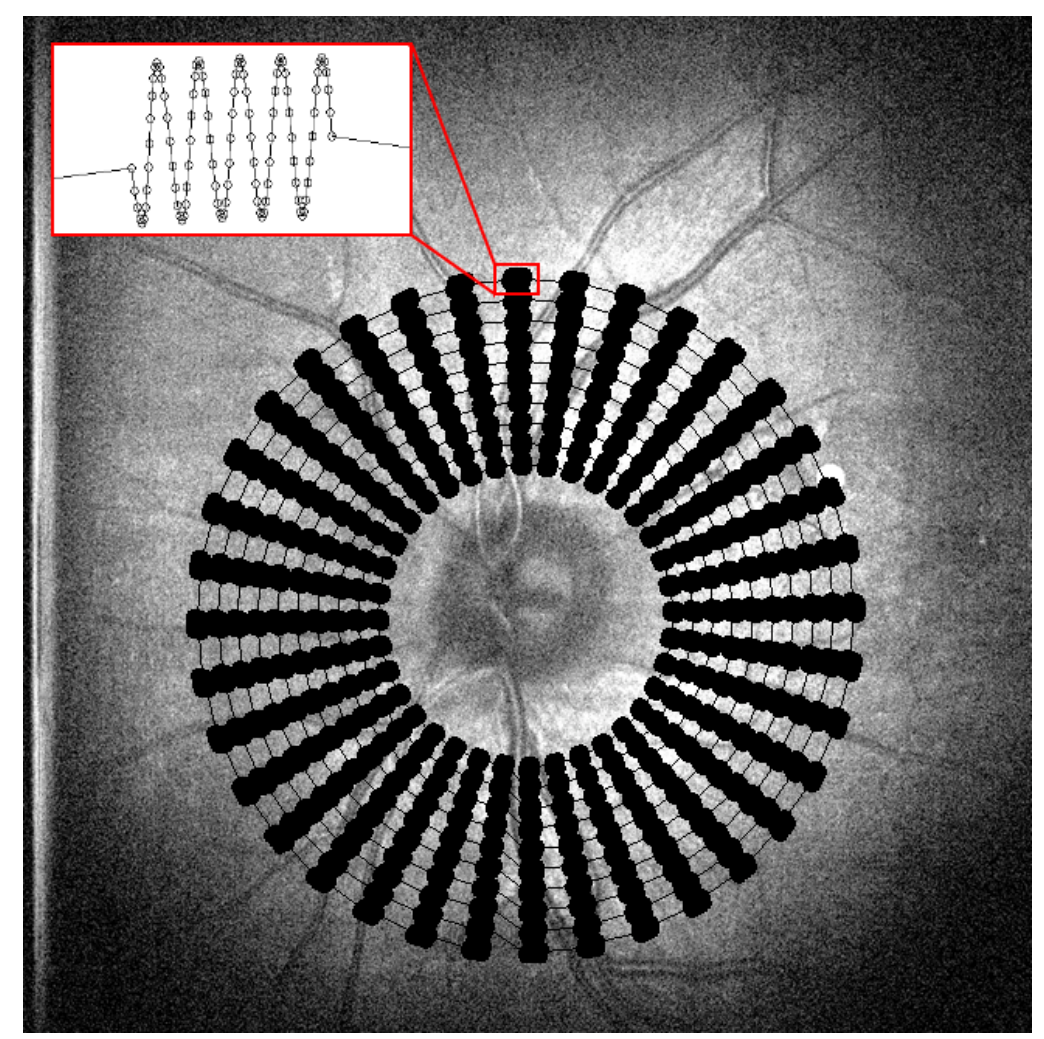

Figure 1: Scanning pattern of a clustered ring scan. The scanning pattern of each cluster is shown in the upper-left corner. Each circle represents an A-scan location.

\subsection{Intensity-based estimate of RNFL boundaries}

RNFL anterior and posterior boundaries are first estimated from the recorded OCT intensity image. Fig. 2(a) shows an example of a B-scan intensity image derived from a clustered ring scan. For each cluster, the intensity profiles of 100 Alines are averaged to suppress speckle noise and improve signal-to-noise ratio (SNR), forming a cluster-averaged intensity image as shown in Fig 2(b). Each averaged A-line of the cluster-averaged image is then processed with a smoothing filter to further reduce speckle noise. A threshold is applied to the averaged and filtered A-line to estimate locations of anterior and posterior RNFL boundaries. Finally, the RNFL segmentation is visually inspected and any misdetected boundaries are manually corrected. The results of intensity-based RNFL segmentation are shown in Fig. 2(c).

\subsection{Polarimetric data processing}

The polarimetric data processing procedure was described previously ${ }^{5}$. Briefly, the horizontal and vertical component of interference fringe signals $\left(E_{h}(z), E_{v}(z)\right)$ and the relative phase retardation between the two orthogonal polarization states $\left(\varphi_{\text {diff }}(z)\right)$ are first cropped to the anterior and posterior RNFL boundaries. The normalized Stokes vectors $S(z)$, which represent the polarization states at each depth-resolved pixel, are then calculated from $E_{h}(z), E_{v}(z)$ and $\varphi_{\text {diff }}(z)$. On the Poincaré Sphere, each polarization state trajectory of normalized Stokes vectors from different RNFL depths traces a circular arc. The three arcs corresponding to the three incident polarization states rotate about the same optical-axis, and the central angle of the arcs corresponds to double pass phase retardation of the segmented RNFL. RNFL phase retardation, birefringence and optical-axis orientation can be estimated from a multiple-state Levenberg-Marquardt nonlinear fitting algorithm. For each cluster, the asymptotic standard error, or uncertainty of birefringence, is calculated from the nonlinear fitting algorithm as an error measure for the estimated birefringence with a $95 \%$ confidence interval ${ }^{21}$. 

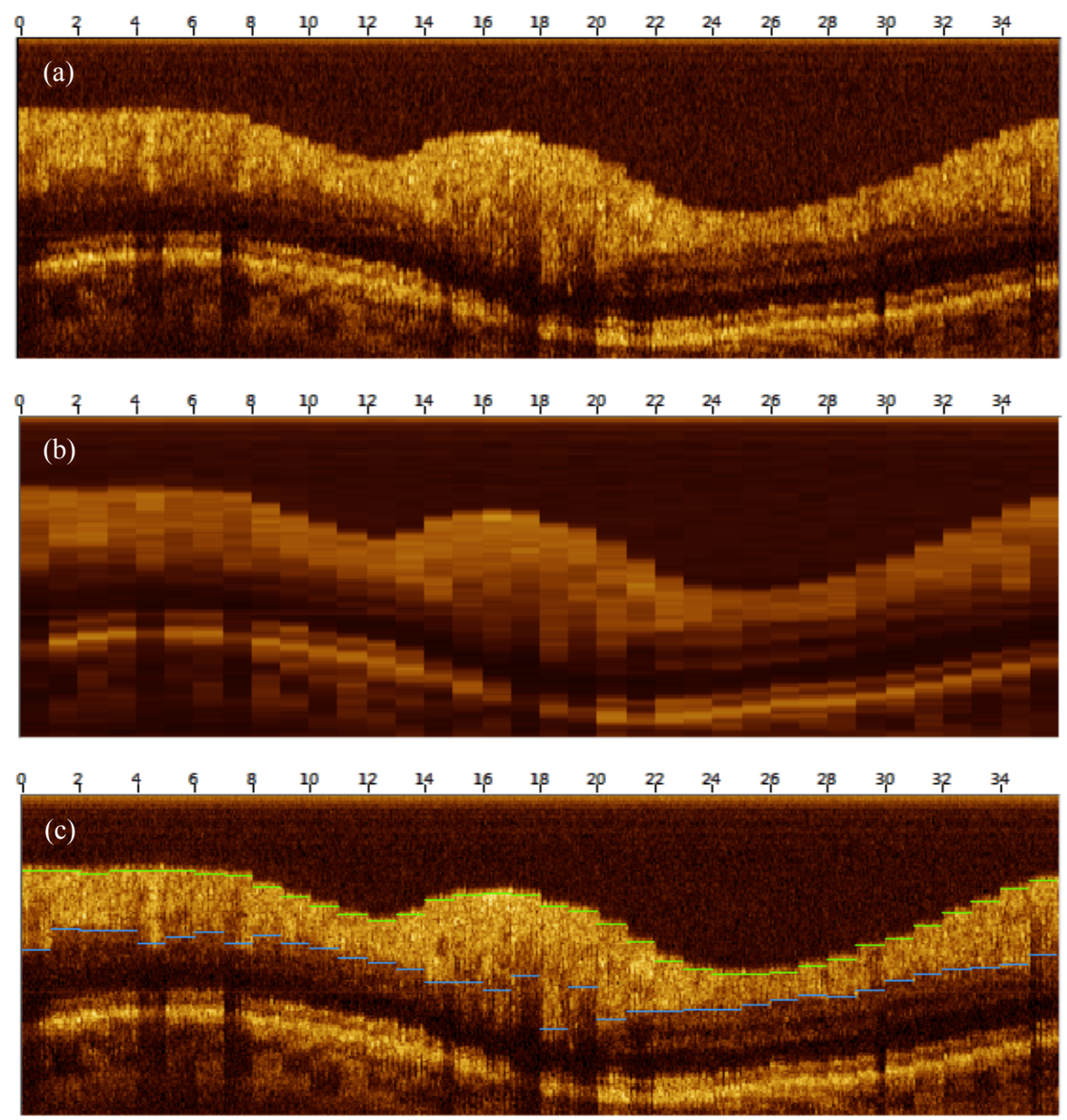

Figure 2. A clustered ring scan acquired about the optic nerve head. Acquired image diameter is $2.3 \mathrm{~mm}$ and consists of 36 clusters of 100 A-lines/cluster. Cluster numbers are marked at the top of the image. (a) Original B-scan intensity image from clustered ring scan. (b) Cluster-averaged intensity image provides improved SNR. (c) B-scan intensity image with intensity-based RNFL boundaries.

\subsection{Optimization of RNFL segmentation based on polarimetric data}

The anterior RNFL boundary is an interface between the vitreous and inner limiting membrane with strong intensity contrast since the refractive index gradient is large. As a result, the RNFL anterior boundary is relatively easy to detect using the intensity image, and in most cases the RNFL anterior boundary is correctly detected using intensity-based segmentation. However the intensity contrast at the RNFL posterior boundary is relatively low because the refractive index gradient from the birefringent RGC axons to their cell bodies is small. To achieve the best estimate of RNFL birefringence, posterior RNFL boundary estimates are refined anatomically with polarimetric information by locally adjusting the RNFL posterior boundary and minimizing the uncertainty of the birefringence estimate. A flow chart of this process is shown in Fig. 3.

To maintain the RNFL posterior boundary in the transition zone of RGC axons and their cell bodies, the search range of the RNFL posterior boundary is constrained to a relatively small depth. In this study, the search range was constrained to 3 pixels above and below (i.e., $\pm 14.07 \mu \mathrm{m}$ ) the intensity-based RNFL posterior boundary, corresponding to the diameter of two RGC cell bodies (around $14 \mu \mathrm{m})^{22}$.

Fig. 4 shows an example of the effects of the RNFL posterior boundary optimization on the Poincare Sphere. The intensity-based segmentation misdetects the RNFL posterior boundary by overestimating three pixels. As a result, the Stokes vector trajectory cropped from the misdetected boundary forms arcs with outlier points near the posterior boundaries. These outliers reduce the fitting quality and increase the uncertainty of estimated birefringence $\left(1.09^{\circ} / 100\right.$ 
$\mu \mathrm{m})$. The optimized RNFL boundary detection successfully removes outliers near the posterior boundary and thus provides better estimates of RNFL birefringence with much lower uncertainty $\left(0.47^{\circ} / 100 \mu \mathrm{m}\right)$.

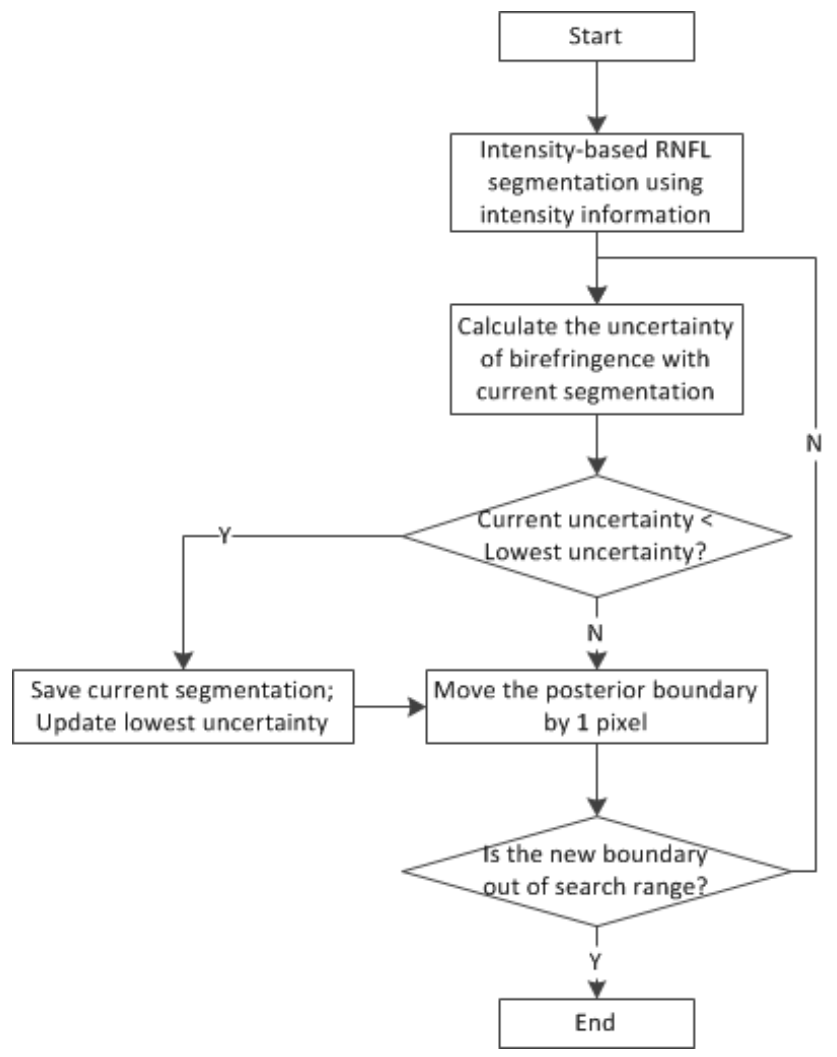

Figure 3. Flow chart of the RNFL boundary optimization algorithm.

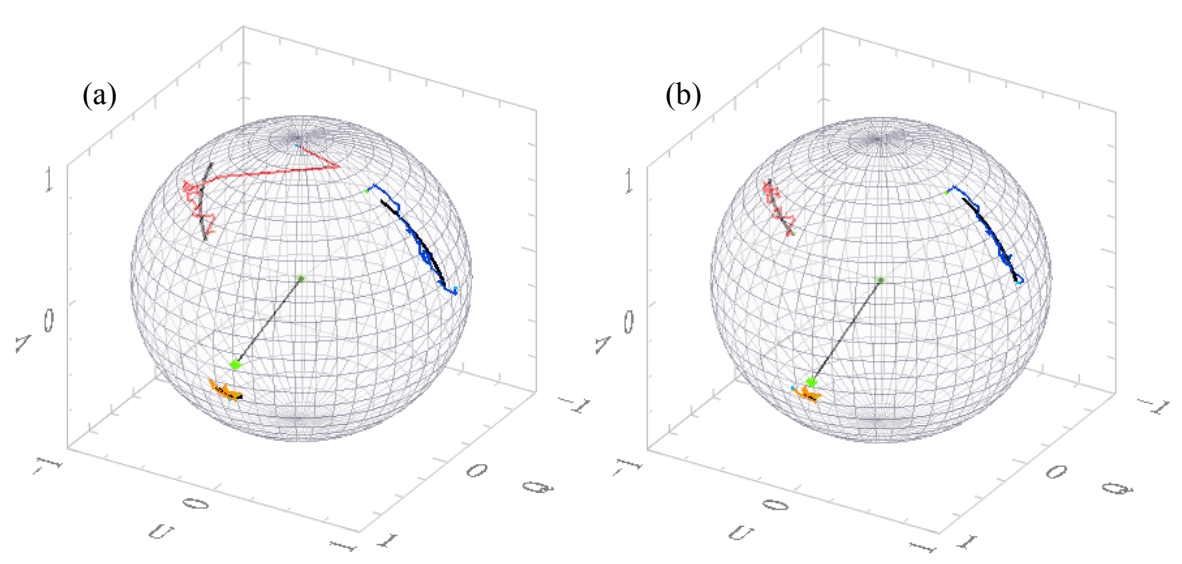

Figure 4. RNFL posterior boundary optimization on the Poincaré Sphere. (a) Intensity-based boundary: RNFL thickness $=$ 36 pixels, uncertainty of birefringence $=1.09^{\circ} / 100 \mu \mathrm{m}$. (b) Optimized boundary: RNFL thickness $=33$ pixels, uncertainty of birefringence $=0.47^{\circ} / 100 \mu \mathrm{m}$.

\section{RESULTS}

Discrete clustered maps of uncertainty, thickness, birefringence (double pass phase retardation per unit depth) and phase retardation created with both intensity-based and optimized RNFL boundaries are shown in Fig. 5. Blood vessel patterns segmented from a raster scan recorded immediately before acquisition of the clustered data are superimposed on the clustered maps. 

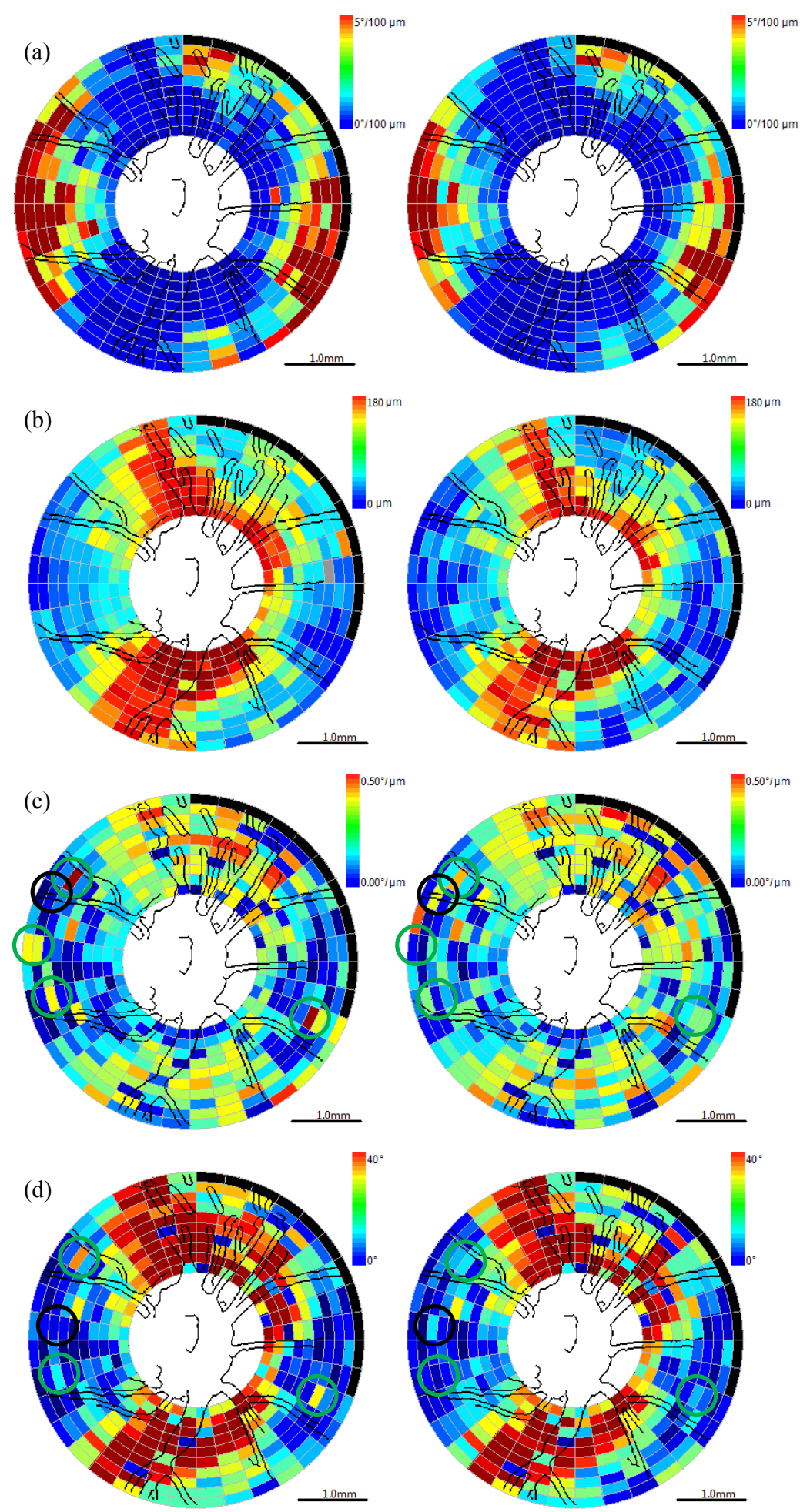

Figure 5. Clustered maps created with intensity-based (left column) and optimized RNFL (right column) boundaries. Unprocessable clusters due to inappropriate operator settings during image acquisition are marked in black. (a) Uncertainty of birefringence. (b) Thickness. (c) Birefringence (double pass phase retardation per unit depth). (d) Phase retardation. Green circles in (c) and (d) mark outlier clusters removed by RNFL boundary optimization, while black circles mark the few new outliers produced by optimized RNFL segmentation. 
As shown in Fig. 5(a), the optimized boundary minimizes the uncertainty of the RNFL birefringence estimate compared with the intensity-based RNFL boundary. Although the optimized boundary is confined within a relatively small transition zone near the intensity-based boundary, RNFL thickness calculated from the intensity-based boundary (average thickness $107.60 \mu \mathrm{m}$ ) is generally greater than the optimized boundary results (average thickness $98.77 \mu \mathrm{m}$ ), as shown in Fig. 5(b). This result suggests that an intensity-based RNFL boundary detection tends to overestimate RNFL thickness, possibly due to a limitation of simple thresholding.

We evaluate the improvement of RNFL birefringence estimate with the proposed RNFL segmentation by assessing the spatial smoothness of RNFL birefringence and phase retardation. Due to the spatial continuity of RNFL physiology, changes in RNFL birefringence and phase retardation between nearby regions of the RNFL are expected to be gradual and smooth. Although the sampling pattern of the clustered ring scan is discrete, the local variation in RNFL birefringence and phase retardation maps should be reasonably small. In the RNFL birefringence (Fig. 5(c)) and phase retardation (Fig. 5(d)) maps values of discontinuous outlier clusters (marked with green circles in Fig. 5 (c) and (d)) are suspected to be inaccurate. Many of these outliers show less variation after the uncertainty-based RNFL boundary optimization. Although the optimized boundary also produces a few new discontinuous clusters (marked with black circles in Fig. 5(c) and (d)) in birefringence and phase retardation maps, the optimized boundary generally provides smoother RNFL birefringence and phase retardation maps.

To evaluate smoothness of RNFL birefringence and phase retardation maps quantitatively, we utilize the local standard deviation of birefringence and phase retardation as a measure of their smoothness. In RNFL birefringence and phase retardation maps, the standard deviation among each cluster and its four neighbors is calculated and plotted in the local standard deviation maps in Fig. 6. For clusters in the innermost and outermost rings, the local standard deviation is calculated with respect to the cluster of interest and its three available neighbors. From Fig. 6 it is clear that the local standard deviation of both RNFL birefringence and phase retardation decrease after the proposed RNFL boundary optimization. The average local standard deviations of RNFL birefringence and phase retardation calculated from intensity-based and optimized RNFL segmentations are given in Table 1. A paired t-test is used to compare the local standard deviation of the intensity-based and optimized segmentation methods. The null hypothesis of the paired t-test is that the mean of the paired differences in the local standard deviation calculated from intensity-based and optimized RNFL segmentations is zero. The paired t-test shows that the decreases in the local standard deviation of birefringence and phase retardation are both statistically significant $\left(p=0.0040\right.$ for birefringence and $p=3.48 \times 10^{-7}$ for phase retardation). The results suggest that RNFL birefringence and phase retardation calculated from the optimized RNFL boundary are spatially smoother than those calculated from the intensity-based boundary. Thus, we conclude that the proposed RNFL segmentation approach provides a better estimate of RNFL birefringence and phase retardation than traditional intensity-based segmentation results.

\section{DISCUSSION}

To the authors' knowledge, this is the first published study of segmenting RNFL from PSOCT images utilizing both intensity and polarimetric information. Many aspects in the current procedure can be improved to achieve better segmentation results and higher performance. For example, currently we adopt a fairly simple approach of estimating RNFL boundary from OCT intensity images. Although the current approach is computationally efficient, it tends to overestimate RNFL thickness as previously mentioned and sometimes it misdetects the RNFL boundary (especially on low quality images). Multiple sophisticated intensity-based RNFL boundary detection algorithms are available and are expected to provide better estimation of intensity-based RNFL boundaries than our current implementation ${ }^{14-19}$.

Another potential problem of the proposed approach is the relatively long processing time. The proposed approach requires running the three-state Levenberg-Marquardt nonlinear fitting algorithm - which involves inversion of a $9 \times 9$ matrix and is computationally intensive - for multiple times at every cluster to find the local minimum of birefringence uncertainty. The whole process requires 15 - 20 minutes for a complete clustered ring scan data set with 360 clusters on an Intel Core 2 Quad CPU computer. However, the performance of the algorithm can be dramatically improved with software coding improvements and utilization of a graphics processing unit (GPU) or field programmable gate array (FPGA) implementation.

In this study, we evaluate the improvement of RNFL birefringence and phase retardation estimation by assessing their local smoothness. Although we argued this is a valid criteria with a reasonable physical assumption that RNFL birefringence and phase retardation is spatially continuous, better approaches of assessing how the proposed algorithm 
improves birefringence and phase retardation estimation may be applied. For example, a low-birefringent material with known birefringence could serve as gold standard. More studies are required to further evaluate the performance of the proposed RNFL segmentation strategy.
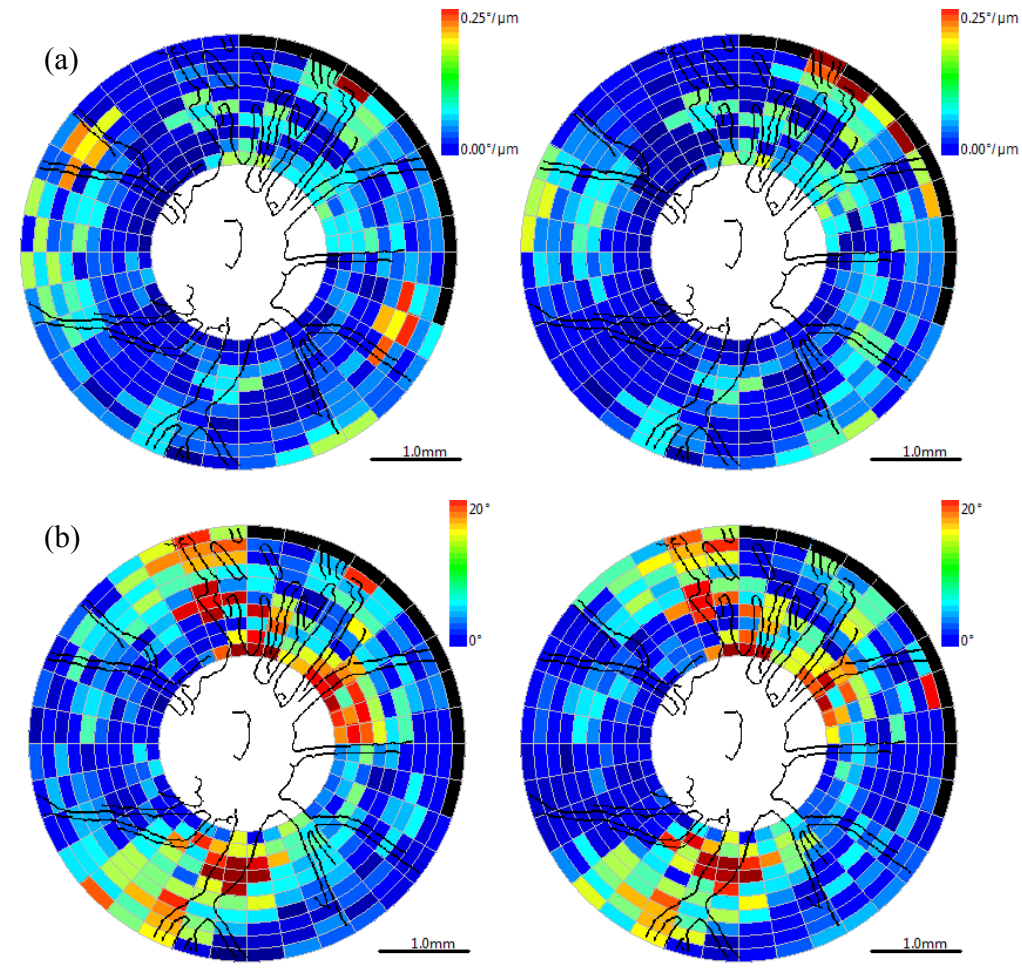

Figure 6. Local standard deviation maps created with intensity-based (left column) and optimized (right column) RNFL boundaries. Unprocessable clusters due to inappropriate operator settings during image acquisition are marked in black. (a) Birefringence. (b) Phase retardation.

Table 1. Comparison of average local standard deviations of birefringence and phase retardation calculated from intensity-based and optimized RNFL segmentations.

\begin{tabular}{|c|c|c|}
\hline & $\begin{array}{c}\text { Average local standard deviation } \\
\text { of birefringence }(\boldsymbol{\%} / \mathbf{1 0 0} \boldsymbol{\mu m} \mathbf{m})\end{array}$ & $\begin{array}{c}\text { Average local standard deviation } \\
\left.\text { of phase retardation } \boldsymbol{(}^{\circ}\right)\end{array}$ \\
\hline Intensity-based segmentation & 7.54 & 8.37 \\
\hline Optimized segmentation & 7.05 & 7.76 \\
\hline Paired t-test $\boldsymbol{p}$-value & 0.0040 & $3.48 \times 10^{-7}$ \\
\hline
\end{tabular}

\section{CONCLUSION}

We propose a two-step RNFL segmentation method for clustered ring scan images acquired with a SS-PSOCT system. The RNFL segmentation method utilizes both intensity and polarimetric information to achieve optimized RNFL boundary detection for estimation of birefringence. RNFL anterior and posterior boundaries are first detected from the intensity image. RNFL birefringence is estimated from the intensity-based RNFL segmentation with a multiple-state Levenberg-Marquardt nonlinear fitting algorithm. Optimized RNFL segmentation is achieved by minimizing the Levenberg-Marquardt uncertainty of RNFL birefringence while locally adjusting the posterior RNFL boundary. The proposed RNFL segmentation approach provides an optimized RNFL segmentation with lowest achievable birefringence uncertainty, and optimizes birefringence and phase retardation measurement. Clinical results from a healthy volunteer suggest that the proposed segmentation method estimates phase retardation in the RNFL with lower uncertainty and higher continuity than traditional intensity-based approaches. 


\section{ACKNOWLEDGMENT}

This research is sponsored by the National Institutes of Health (R01 EY016462-02).

\section{REFERENCES}

[1] Huang, D., Swanson, E. A., Lin, C. P. et al. "Optical coherence tomography," Science 254, 1178 (1991).

[2] Hee, M. R., Huang, D., Swanson, E. A. \& Fujimoto, J. G. "Polarization-sensitive low-coherence reflectometer for birefringence characterization and ranging," Journal of the Optical Society of America B 9, 903-908 (1992).

[3] De Boer, J. F., Milner, T. E., van Gemert, M. J. C. \& Nelson, J. S. "Two-dimensional birefringence imaging in biological tissue by polarization-sensitive optical coherence tomography," Optics Letters 22, 934-936 (1997).

[4] Saxer, C. E., de Boer, J. F., Park, B. H. et al. "High-speed fiber based polarization-sensitive optical coherence tomography of in vivo human skin," Optics Letters 25, 1355-1357 (2000).

[5] Elmaanaoui, B., Wang, B., Dwelle, J. C. et al. "Birefringence measurement of the retinal nerve fiber layer by swept source polarization sensitive optical coherence tomography," Optics Express 19, 10252-10268 (2011).

[6] Cense, B., Chen, T. C., Park, B. H., Pierce, M. C. \& De Boer, J. F. "Thickness and birefringence of healthy retinal nerve fiber layer tissue measured with polarization-sensitive optical coherence tomography," Investigative ophthalmology \& visual science 45, 2606 (2004).

[7] Yamanari, M., Miura, M., Makita, S., Yatagai, T. \& Yasuno, Y. "Phase retardation measurement of retinal nerve fiber layer by polarization-sensitive spectral-domain optical coherence tomography and scanning laser polarimetry," Journal of biomedical optics 13, 014013 (2008).

[8] Götzinger, E., Pircher, M., Baumann, B. et al. "Retinal nerve fiber layer birefringence evaluated with polarization sensitive spectral domain oct and scanning laser polarimetry: A comparison," Journal of Biophotonics 1, 129-139 (2008).

[9] Mujat, M., Park, B. H., Cense, B., Chen, T. C. \& de Boer, J. F. "Autocalibration of spectral-domain optical coherence tomography spectrometers for in vivo quantitative retinal nerve fiber layer birefringence determination," Journal of biomedical optics 12, 041205 (2007).

[10] Huang, X. R. \& Knighton, R. W. "Microtubules contribute to the birefringence of the retinal nerve fiber layer," Investigative ophthalmology \& visual science 46, 4588 (2005).

[11] Park, J., Kemp, N. J., Zaatari, H. N., Rylander III, H. G. \& Milner, T. E. "Differential geometry of normalized stokes vector trajectories in anisotropic media," JOSA A 23, 679-690 (2006).

[12] Park, J., Kemp, N. J., Rylander, H. G. \& Milner, T. E. "Complex polarization ratio to determine polarization properties of anisotropic tissue using polarization-sensitive optical coherence tomography," Optics Express 17, 13402-13417 (2009).

[13] Pocock, G. M., Aranibar, R. G., Kemp, N. J. et al. "The relationship between retinal ganglion cell axon constituents and retinal nerve fiber layer birefringence in the primate," Investigative ophthalmology \& visual science 50, 5238 (2009).

[14] Mujat, M., Chan, R., Cense, B. et al. "Retinal nerve fiber layer thickness map determined from optical coherence tomography images," Optics Express 13, 9480-9491 (2005).

[15] Garvin, M. K., Abràmoff, M. D., Kardon, R. et al. "Intraretinal layer segmentation of macular optical coherence tomography images using optimal 3-d graph search," IEEE Transactions on Medical Imaging 27, 1495-1505 (2008).

[16] Paranjape, A. S., Elmaanaoui, B., Dewelle, J., Rylander, H. G. \& Milner, T. E. "Automated method for rnfl segmentation in spectral domain oct," Proc. SPIE 6848, 68480N (2008).

[17] Mishra, A., Wong, A., Bizheva, K. \& Clausi, D. A. "Intra-retinal layer segmentation in optical coherence tomography images," Optics Express 17, 23719-23728 (2009).

[18] Kajić , V., Považay, B., Hermann, B. et al. "Robust segmentation of intraretinal layers in the normal human fovea using a novel statistical model based on texture and shape analysis," Optics Express 18, 14730-14744 (2010).

[19] Mayer, M. A., Hornegger, J., Mardin, C. Y. \& Tornow, R. P. "Retinal nerve fiber layer segmentation on fd-oct scans of normal subjects and glaucoma patients," Biomedical Optics Express 1, 1358-1383 (2010). 
[20] Kemp, N. J., Park, J., Zaatari, H. N., Rylander, H. G. \& Milner, T. E. "High-sensitivity determination of birefringence in turbid media with enhanced polarization-sensitive optical coherence tomography," Journal of the Optical Society of America A 22, 552-560 (2005).

[21] Marquardt, D. W. "An algorithm for least-squares estimation of nonlinear parameters," Journal of the society for Industrial and Applied Mathematics 11, 431-441 (1963).

[22] Glovinsky, Y., Quigley, H. \& Dunkelberger, G. "Retinal ganglion cell loss is size dependent in experimental glaucoma," Investigative ophthalmology \& visual science 32, 484 (1991). 